Article

\title{
Flexible Thermoelectric Composite Films of Polypyrrole Nanotubes Coated Paper
}

\author{
Jun $\mathrm{Li}^{1}$, Yong Du ${ }^{1, *}$ (D), Runping Jia ${ }^{1}$, Jiayue $\mathrm{Xu}^{1, *}$ and Shirley Z. Shen ${ }^{2}$ \\ 1 School of Materials Science and Engineering, Shanghai Institute of Technology, Shanghai 201418, China; \\ 156081106@mail.sit.edu.cn (J.L.); Jrpchem@126.com (R.J.) \\ 2 CSIRO Manufacturing, Private Bag 10, Clayton South, VIC 3169, Australia; shirley.shen@csiro.au \\ * Correspondence: ydu@sit.edu.cn (Y.D.); xujiayue@sit.edu.cn (J.X.); Tel.: +86-21-6087-3581 (Y.D. \& J.X.) \\ Academic Editor: Norbert M. Nemes \\ Received: 16 October 2017; Accepted: 13 November 2017; Published: 24 November 2017
}

\begin{abstract}
Flexible thermoelectric composite films of polypyrrole (PPy) nanotubes coated paper were fabricated by an in-situ polymerization procedure using methyl orange as a template and paper as the substrate for the first time. Both the electrical conductivity and Seebeck coefficient of the polypyrrole nanotubes coated paper composite films have been enhanced (from $\sim 0.048 \mathrm{~S} / \mathrm{cm}$ to $\sim 0.068 \mathrm{~S} / \mathrm{cm}$ and from $\sim 5.34 \mu \mathrm{V} / \mathrm{K}$ to $\sim 8.21 \mu \mathrm{V} / \mathrm{K}$ for the average value for three measurements, respectively) as the temperature increased from $\sim 300 \mathrm{~K}$ to $\sim 370 \mathrm{~K}$, which lead to the same trend of the power factor. The thermal conductivity of the polypyrrole nanotubes coated composite films was very low $\left(\sim 0.1275 \mathrm{~W} \cdot \mathrm{m}^{-1} \cdot \mathrm{K}^{-1}\right.$ at $\left.\sim 300 \mathrm{~K}\right)$, and a highest $\mathrm{ZT}$ (material's dimensionless figure of merit $\left.\left(S^{2} \sigma T / \mathrm{K}\right)\right)$ value of $3.2 \times 10^{-7}$ was obtained at $\sim 370 \mathrm{~K}$.
\end{abstract}

Keywords: PPy nanotubes; coating; flexible; thermoelectric properties

\section{Introduction}

Polypyrrole (PPy) is a potential thermoelectric (TE) material, mainly because it has a low thermal conductivity and high electrical conductivity when doped with suitable dopants. PPy also has many advantages, such as being easy to fabricate, low-density, low-cost, and having good mechanical properties [1]. Therefore, more and more researchers have focused on the TE properties of PPy and its corresponding composites [2-6]. For example, a maximum $Z T$ value $\left(S^{2} \sigma T / \kappa, S\right.$ is the Seebeck coefficient, $\sigma$ is the electrical conductivity, $\mathrm{k}$ is the thermal conductivity, and $T$ is the absolute temperature) of $2.8 \times 10^{-3}$ at room temperature (RT) was achieved for the PPy/graphene nanosheet composites with a weight ratio of pyrrole monomer to graphene nanosheets of 1:0.4 [2]; a maximum power factor $\left(S^{2} \sigma\right)$ of $2.079 \mu \mathrm{W} \cdot \mathrm{m}^{-1} \cdot \mathrm{K}^{-2}$ was obtained at RT for multi-walled carbon nanotube/PPy composites with $20 \mathrm{wt} \%$ multi-walled carbon nanotubes [3], and a power factor of $52.5 \mu \mathrm{W} \cdot \mathrm{m}^{-1} \cdot \mathrm{K}^{-2}$ was achieved by a PPy/graphene/polyaniline ternary nanocomposite with $32 \mathrm{wt} \%$ graphene at $90{ }^{\circ} \mathrm{C}$ [7]. Most recently, a ZT value of 0.0043 was calculated along its chain direction of a crystalline PPy-PF 6 using the first-principles method [1].

As a 1D material, polypyrrole nanotubes (PPy-NTs) have a higher electrical conductivity when compared to PPy nanoparticles [8,9]; therefore, it is beneficial to enhancing its TE properties. For example, a ZT value of $5.71 \times 10^{-4}$ (power factor of $0.31 \mu \mathrm{W} \cdot \mathrm{m}^{-1} \cdot \mathrm{K}^{-2}$ ) at $310 \mathrm{~K}$ was reported for the PPy-NT films [10]. Furthermore, Misra et al. [11] prepared PPy-NTs and then treatment with various dopants, e.g., $\mathrm{HCl}$, p-toluene-sulphonic acid monohydrate (ToS), and tetrabutyl ammonium hexaflurophosphate $\left(\mathrm{PF}_{6}\right)$. A highest $\mathrm{ZT}$ value of $\sim 3.4 \times 10^{-3}$ (power factor of $0.08 \mu \mathrm{W} \cdot \mathrm{m}^{-1} \cdot \mathrm{K}^{-2}$ ) was achieved at $380 \mathrm{~K}$ for the $\mathrm{PF}_{6}$ doping of PPy-NTs. During chemical oxidative polymerization, the anions (e.g., chloride, sulfate, phosphate, oxalate, and acetate ion) of the dopants can be incorporated along a polymer backbone $[12,13]$. These studies show that PPy-NT has a potential to be used as a TE material. 
Paper is a suitable substrate for flexible TE materials, due to its low density, low cost, and flexibility. Many researchers use paper as a substrate to prepare flexible TE materials. Jiang et al. [14] prepared poly(3,4-ethylenedioxythiophene):poly(styrenesulfonate) (PEDOT:PSS)/paper composite films, and a maximum $Z T$ value of $5.5 \times 10^{-3}$ was obtained at $300 \mathrm{~K}$. Wei et al. [15] fabricated the TE modules by screen printing PEDOT:PSS on paper.

If a suitable method can be applied to coat paper with PPy-NTs, it will endow paper with TE properties. However, so far, to the best of our knowledge, no research on the TE properties of PPy-NT/paper composite films has been reported. In this work, flexible PPy-NT/paper composite films was prepared via an in-situ chemical polymerization procedure using methyl orange (MO) as a template. The composition, morphology, and TE properties of the PPy-NT/paper composite films have been investigated.

\section{Materials and Methods}

\subsection{Materials}

Pyrrole monomer (Chemical grade) was purchased from Sigma-Aldrich (Shanghai, China). Methyl orange (reagent grade) was purchased from Adamas Reagent Co., Ltd. (Shanghai, China). $\mathrm{FeCl}_{3} \cdot 6 \mathrm{H}_{2} \mathrm{O}$ (analytical reagent) and absolute ethanol (reagent grade) were purchased from Sinopharm Chemical Reagent Co., Ltd. (Shanghai, China). Paper was purchased from Zhanjiang Chenming Paper Co., Ltd. (Zhanjiang, China, the density and thickness is $70 \mathrm{~g} / \mathrm{m}^{2}$ and $96 \mu \mathrm{m}$, respectively). All the materials were used without further treatment or purification.

\subsection{Preparation of the Flexible PPy-NT/Paper Composite Films}

$0.56 \mathrm{~mL}$ pyrrole monomer and $0.49 \mathrm{~g}$ methyl orange (MO) were dissolved in $150 \mathrm{~mL}$ of deionized water (Solution A), then a piece of common printing paper $(3 \mathrm{~cm} \times 3 \mathrm{~cm})$ was soaked in Solution A, and stirring continued for $30 \mathrm{~min} .8 .72 \mathrm{~g} \mathrm{FeCl}_{3} \cdot 6 \mathrm{H}_{2} \mathrm{O}$ dissolved in $100 \mathrm{~mL}$ of deionized water (Solution B) was then added to Solution A and constantly stirred for $8 \mathrm{~h}$ at RT. After oxidization $\mathrm{FeCl}_{3} \cdot 6 \mathrm{H}_{2} \mathrm{O}$ was added into the solution, the acidity of the solution was enhanced due to the hydrolysis of $\mathrm{FeCl}_{3} \cdot 6 \mathrm{H}_{2} \mathrm{O}$. As a result, the salt form of $\mathrm{MO}$ with high water solubility was changed into the acid form of MO with poor water solubility, and then $1 \mathrm{D}$ templates were formed for the polymerization of pyrrole monomer on the surface of paper $[8,16]$. After that, the PPy-NT/paper composite film was taken out by using a pair of tweezers and washed with deionized water and absolute ethanol three times, respectively. The washed composite film was dried at $60^{\circ} \mathrm{C}$ for $12 \mathrm{~h}$, and then soaked in $1 \mathrm{~mol} / \mathrm{L}$ hydrochloride $(\mathrm{HCl})$ for $12 \mathrm{~h}$. Finally, the composite film was dried at $60^{\circ} \mathrm{C}$ for $12 \mathrm{~h}$. For a comparison, PPy-NTs were also prepared using the same procedures as above, but without a piece of paper. Figure 1 illustrates the procedure for the preparation of flexible PPy-NT/paper composite films.

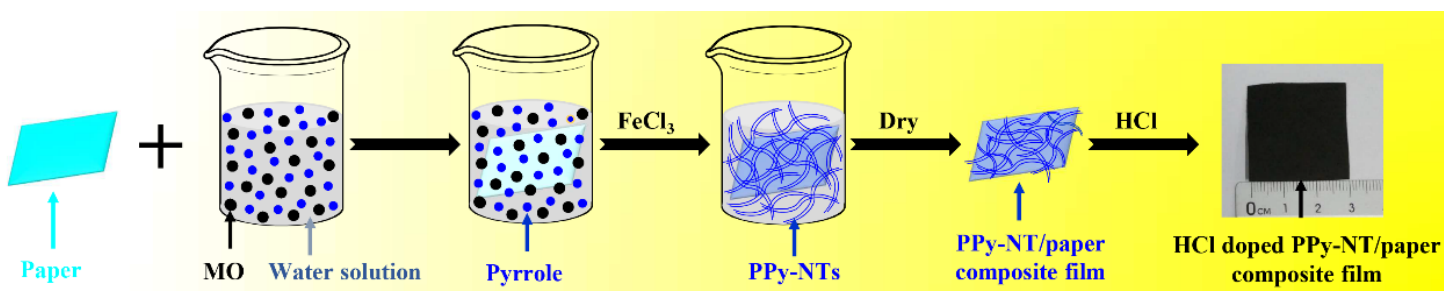

Figure 1. The fabrication process of the polypyrole nanotube (PPy-NT)/paper composite films.

\subsection{Characterizations}

The phase composition and morphology of the samples were characterized using X-ray photoelectron spectroscopic (XPS, PHI 5000 VersaProbe, ULVAC-PHI, Chigasaki, Japan), scanning electron microscopy (SEM, Philips XL 30 FEG, Philips, Eindhoven, The Netherlands), and 
transmission electron microscopy (TEM, CM200-FEG, Philips, Eindhoven, The Netherlands), respectively. In-plane electrical conductivity and Seebeck coefficient were measured simultaneously in a vacuum atmosphere from 300 to $370 \mathrm{~K}$ on a MRS-3L thin-film thermoelectric test system (Wuhan Giant Instrument Technology Co., Ltd., Wuhan, China). Out-plane thermal conductivities of the samples were measured by a transient hot-wire method at RT (TC3000E thermal conductivity meter, Xiatech Electronics Co., Xi'an, China).

\section{Results and Discussion}

Figure 2a-c shows the SEM images of the paper, PPy-NTs, and PPy-NT/paper composite films, respectively. Figure $2 \mathrm{~d}$ shows the TEM image of PPy-NTs. The diameter of the prepared PPy-NTs is $70 \mathrm{~nm}$ to $150 \mathrm{~nm}$, and the lengths are in the $\mu \mathrm{m}$-range. The PPy-NTs are found to be uniformly coated on the surface of paper. Figure 2e shows the SEM image of the fracture surface of the PPy-NT/paper composite films. The film thickness measured based on the Figure 2e is $\sim 124 \mu \mathrm{m}$, which is slightly thicker than that of the common printing paper $(110 \mu \mathrm{m})$. This is mainly because the surface of paper was coated by PPy-NTs. After the PPy-NT coating, the color of paper changed from white to black. The prepared PPy-NT/paper composites have a good flexibility (Figure 3a-d).

The XPS of the PPy-NTs coated paper is shown in Figure 4. For the paper, it mainly contained C and O, whereas for the PPy-NTs and PPy-NTs-coated paper films, $\mathrm{N}$ has been observed, which indicates the PPy-NTs were successfully coating on the paper. Note that $\mathrm{Cl}$ came from $\mathrm{HCl}$, because the composite film was doped with $\mathrm{HCl}$.

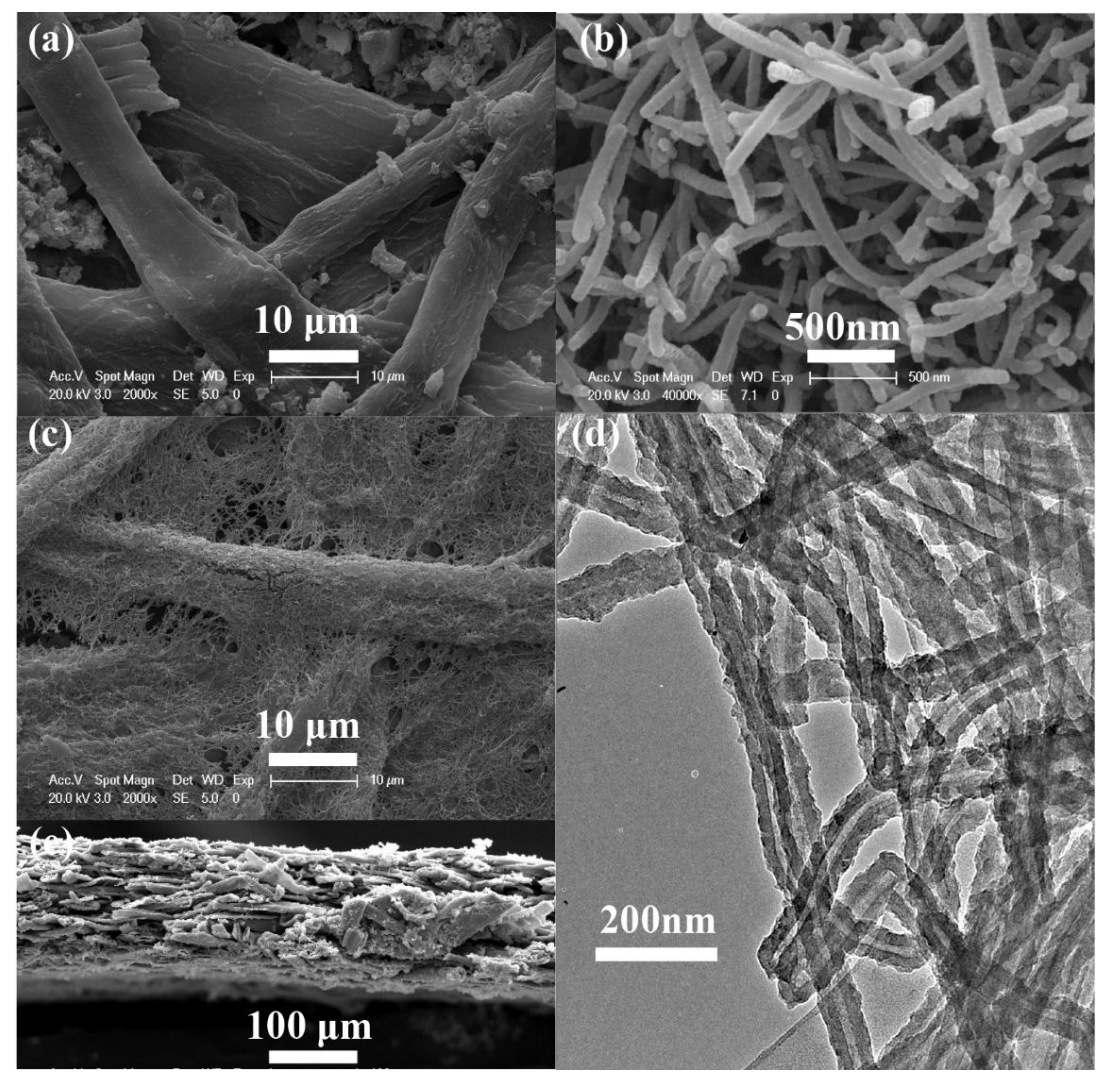

Figure 2. SEM images of (a) paper, (b) PPy-NTs, and (c) PPy-NT/paper composite film. TEM image of (d) PPy-NTs and (e) fracture surface of PPy-NT/paper composite film. 


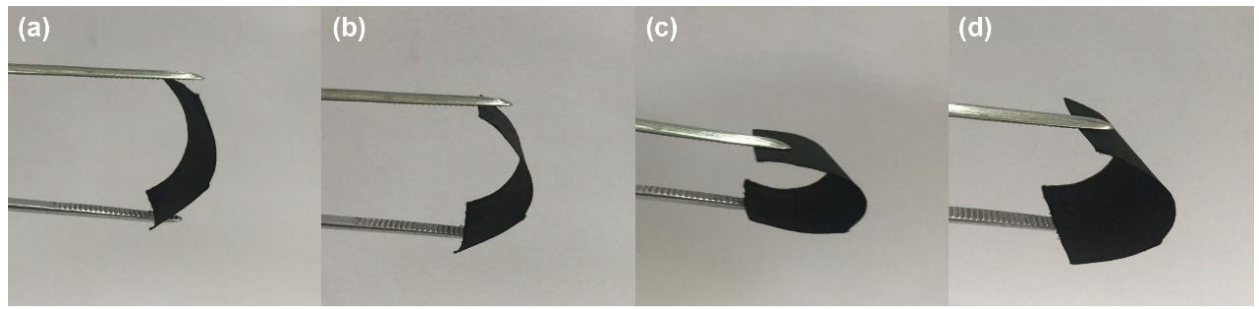

Figure 3. The flexible display digital photos of the PPy-NT/paper composite film.
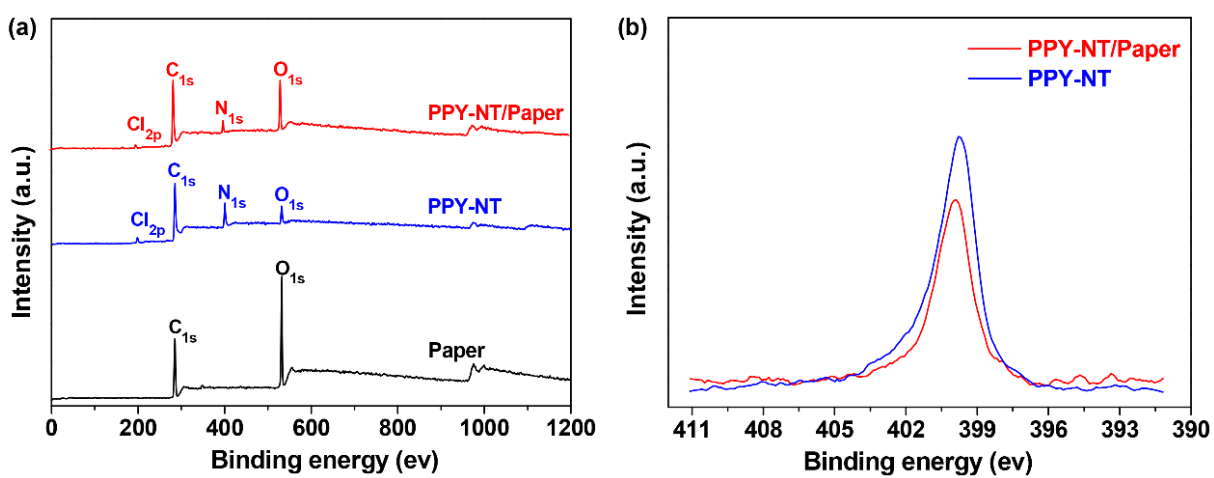

Figure 4. (a) XPS spectra of wide region spectroscopy of paper, PPy-NTs, and PPy-NT/paper composite film; (b) XPS spectra of $\mathrm{N}_{1 S}$ of PPy-NTs and PPy-NT/paper composite film.

The electrical conductivity, Seebeck coefficient, and power factor of the PPy-NT/paper are shown in Figure 5. The sample was cycled three times in the measured temperature range from $\sim 300 \mathrm{~K}$ to $\sim 370 \mathrm{~K}$. Both the electrical conductivity and Seebeck coefficient are very stable. The electrical conductivity of the composite films increased slowly from $\sim 0.048 \mathrm{~S} / \mathrm{cm}$ to $\sim 0.068 \mathrm{~S} / \mathrm{cm}$ (average value for three measurements) as the temperature increased from $\sim 300 \mathrm{~K}$ to $\sim 370 \mathrm{~K}$. However, this value is still lower than that of the PPy particle $(3.98 \mathrm{~S} / \mathrm{cm})$ [2], PPy-NT film $(9.81 \mathrm{~S} / \mathrm{cm})$ [10], HCl doped PPy-NTs $(10.6 \mathrm{~S} / \mathrm{cm})$ [11], or PF6 doped PPy-NTs $(2.4 \mathrm{~S} / \mathrm{cm})$ [11]. It is mainly due to the electrical isolation characteristic of paper [17]. The Seebeck coefficient also increased slowly from $\sim 5.34 \mu \mathrm{V} / \mathrm{K}$ to $\sim 8.21 \mu \mathrm{V} / \mathrm{K}$ (average value for three measurements) as the temperature increased in the measured temperature range. The positive value of the Seebeck coefficient means PPy-NT/paper composite film is a p-type TE material. This value is lower than that of reported elsewhere, such as PPy particle $(9.72 \mu \mathrm{V} / \mathrm{K})$ [2], PPy-NT film $(17.68 \mu \mathrm{V} / \mathrm{K})$ [10], $\mathrm{HCl}$ doped PPy-NTs $(11.4 \mu \mathrm{V} / \mathrm{K})$ [11], and $\mathrm{PF}_{6}$ doped PPy-NTs $(18.5 \mu \mathrm{V} / \mathrm{K})$ as well [11].

The power factor of PPy-NT/paper composite films also increased as the temperature increased from $\sim 300 \mathrm{~K}$ to $\sim 370 \mathrm{~K}$, due to the same trend of the electrical conductivity and Seebeck coefficient of the composite films. A high power factor of $4.56 \times 10^{-4} \mu \mathrm{W} \cdot \mathrm{m}^{-1} \cdot \mathrm{K}^{-2}$ (average value for three measurements) has been achieved at $\sim 370 \mathrm{~K}$. The thermal conductivity of the composite films is only $0.1275 \mathrm{~W} \cdot \mathrm{m}^{-1} \cdot \mathrm{K}^{-1}$ at $300 \mathrm{~K}$, which is much lower than that of inorganic materials, such as SnSe crystal $\left(0.46-0.7 \mathrm{~W} \cdot \mathrm{m}^{-1} \cdot \mathrm{K}^{-1}\right)$ [18], and PPy-NT films $\left(0.17 \mathrm{~W} \cdot \mathrm{m}^{-1} \cdot \mathrm{K}^{-1}\right)$ [10]. The thermal conductivity is the sum of electronic contribution and phonon contribution [14], and the electronic contribution is directly proportional to the electrical conductivity. For the PPy-NT/paper composite films, the electrical conductivity is only $0.048 \mathrm{~S} / \mathrm{cm}$ at $300 \mathrm{~K}$, which may be the main reason of the low thermal conductivity of PPy-NT/paper composite films. The ZT value of $3.2 \times 10^{-7}$ was obtained at $300 \mathrm{~K}$, which is lower than that of the traditional TE materials (such as Bi-Te based alloys with ZT 1 [19]) and common thermocouples (e.g., Fe-constantan thermocouple with ZT $\sim 5 \times 10^{-4}$ [20]). Although this value is too low when compared to inorganic TE materials, e.g., SnSe [18] and BiSbTe [21], and 
conducting polymer, e.g., PEDOT:PSS [22,23], this is still a facile method to endow paper with stable TE properties. The process can be extended to other conducting polymers and substrates.

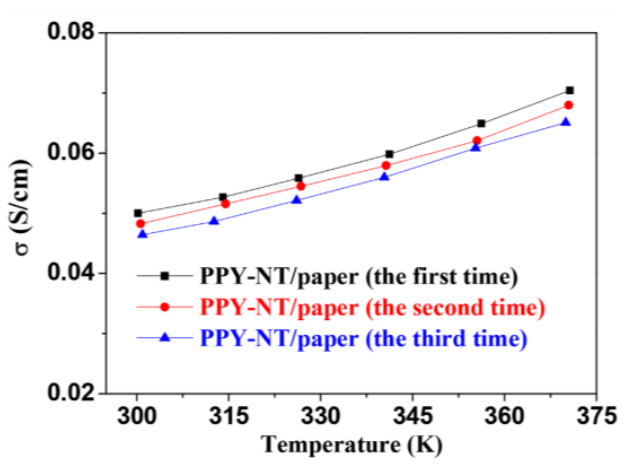

(a)

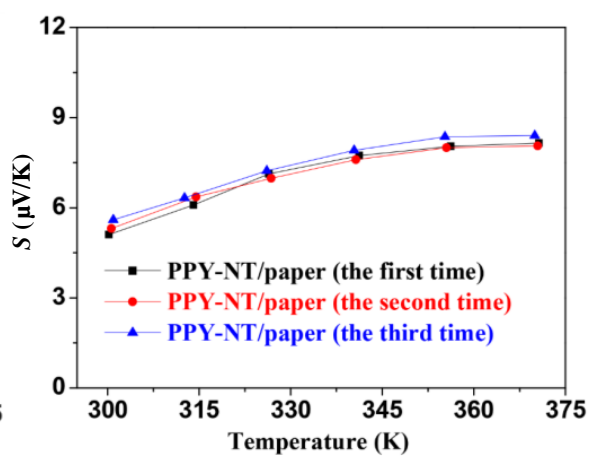

(b)

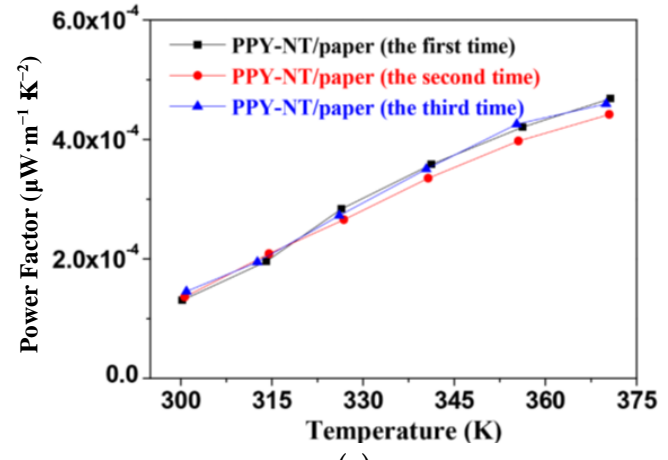

(c)

Figure 5. (a) Electrical conductivity, (b) Seebeck coefficient, and (c) calculated power factor of the PPy-NT/paper composite film.

\section{Conclusions}

Flexible PPy-NT/paper composite thermoelectric films have been prepared by an in situ polymerization procedure using methyl orange as a template and paper as the substrate. The composite films show very stable TE properties in three measurements from $\sim 300 \mathrm{~K}$ to $\sim 370 \mathrm{~K}$. Both the electrical conductivity and Seebeck coefficient of the PPy-NT/paper nanocomposite films were slowly enhanced as the measured temperature increased. The composite film has a low thermal conductivity of $0.1275 \mathrm{~W} \cdot \mathrm{m}^{-1} \cdot \mathrm{K}^{-1}$, and a ZT value of $3.2 \times 10^{-7}$ at $\sim 300 \mathrm{~K}$.

Acknowledgments: This work has been supported by the National Natural Science Foundation of China (61611530550, 61504081), the Program for Professor of Special Appointment (Young Eastern Scholar Program) at Shanghai Institutions of Higher Learning (QD2015039), and the Shanghai Innovation action plan project (17090503600, 15520503400).

Author Contributions: Jun Li performed the experiments, data analysis, and wrote the manuscript. Yong Du designed the experiments and wrote the manuscript. Runping Jia, Shirley Z. Shen, and Jiayue Xu offered valuable discussions in analyses and revised the manuscript.

Conflicts of Interest: The authors declare no conflict of interest.

\section{References}

1. Li, C.; Ma, H.; Tian, Z. Thermoelectric properties of crystalline and amorphous polypyrrole: A computational study. Appl. Therm. Eng. 2017, 111, 1441-1447. [CrossRef]

2. Wang, L.; Liu, F.; Jin, C.; Zhang, T.; Yin, Q. Preparation of polypyrrole/graphene nanosheets composites with enhanced thermoelectric properties. RSC Adv. 2014, 4, 46187-46193. [CrossRef]

3. Wang, J.; Cai, K.; Shen, S.; Yin, J. Preparation and thermoelectric properties of multi-walled carbon nanotubes/polypyrrole composites. Synth. Met. 2014, 195, 132-136. [CrossRef] 
4. Liang, L.; Chen, G.; Guo, C.-Y. Polypyrrole nanostructures and their thermoelectric performance. Mater. Chem. Front. 2017, 1, 380-386. [CrossRef]

5. Liang, L.; Chen, G.; Guo, C.-Y. Enhanced thermoelectric performance by self-assembled layered morphology of polypyrrole nanowire/single-walled carbon nanotube composites. Compos. Sci. Technol. 2016, 129, 130-136. [CrossRef]

6. Han, S.; Zhai, W.; Chen, G.; Wang, X. Morphology and thermoelectric properties of graphene nanosheets enwrapped with polypyrrole. RSC Adv. 2014, 4, 29281-29285. [CrossRef]

7. Wang, Y.; Yang, J.; Wang, L.; Du, K.; Yin, Q.; Yin, Q. Polypyrrole/Graphene/Polyaniline Ternary Nanocomposite with High Thermoelectric Power Factor. ACS Appl. Mater. Interfaces 2017, 9, 20124-20131. [CrossRef] [PubMed]

8. Sapurina, I.; Li, Y.; Alekseeva, E.; Bober, P.; Trchová, M.; Morávková, Z.; Stejskal, J. Polypyrrole nanotubes: The tuning of morphology and conductivity. Polymer 2017, 113, 247-258. [CrossRef]

9. Škodová, J.; Kopecký, D.; Vrňata, M.; Varga, M.; Prokeš, J.; Cieslar, M.; Bober, P.; Stejskal, J. Polypyrrole-silver composites prepared by the reduction of silver ions with polypyrrole nanotubes. Polym. Chem. 2013, 4, 3610-3616. [CrossRef]

10. Wu, J.; Sun, Y.; Pei, W.; Huang, L.; Xu, W.; Zhang, Q. Polypyrrole nanotube film for flexible thermoelectric application. Synth. Met. 2014, 196, 173-177. [CrossRef]

11. Misra, S.; Bharti, M.; Singh, A.; Debnath, A.; Aswal, D.; Hayakawa, Y. Nanostructured polypyrrole: Enhancement in thermoelectric figure of merit through suppression of thermal conductivity. Mater. Res. Express 2017, 4, 085007. [CrossRef]

12. Shaktawat, V.; Sharma, K.; Saxena, N. Structural and electrical characterization of protonic acid doped polypyrrole. J. Ovonic Res. 2010, 6, 239-245.

13. Shaktawat, V.; Jain, N.; Saxena, R.; Saxena, N.; Sharma, T. Electrical conductivity and optical band gap studies of polypyrrole doped with different acids. J. Optoelectron. Adv. Mater. 2007, 9, 2130-2132.

14. Jiang, Q.; Liu, C.; Xu, J.; Lu, B.; Song, H.; Shi, H.; Yao, Y.; Zhang, L. Paper: An effective substrate for the enhancement of thermoelectric properties in PEDOT:PSS. J. Polym. Sci. Part A Polym. Phys. 2014, 52, 737-742. [CrossRef]

15. Wei, Q.; Mukaida, M.; Kirihara, K.; Naitoh, Y.; Ishida, T. Polymer thermoelectric modules screen-printed on paper. RSC Adv. 2014, 4, 28802-28806. [CrossRef]

16. Kopecká, J.; Kopecký, D.; Vrňata, M.; Fitl, P.; Stejskal, J.; Trchová, M.; Bober, P.; Morávková, Z.; Prokeš, J.; Sapurina, I. Polypyrrole nanotubes: Mechanism of formation. RSC Adv. 2014, 4, 1551-1558. [CrossRef]

17. Li, J.; Du, Y.; Jia, R.; Xu, J.; Shen, S. Thermoelectric properties of flexible PEDOT:PSS/polypyrrole/paper nanocomposite films. Materials 2017, 10, 780. [CrossRef] [PubMed]

18. Zhao, L.; Lo, S.; Zhang, Y.; Sun, H.; Tan, G.; Uher, C.; Wolverton, C.; Dravid, V.; Kanatzidis, M. Ultralow thermal conductivity and high thermoelectric figure of merit in SnSe crystals. Nature 2014, 508, 373-377. [CrossRef] [PubMed]

19. Kim, D.; Kim, C.; Heo, S.; Kim, H. Influence of powder morphology on thermoelectric anisotropy of spark-plasma-sintered Bi-Te-based thermoelectric materials. Acta Mater. 2011, 59, 405-411. [CrossRef]

20. Singh, J.; Verma, S. A Comparison of figure of merit for some common thermocouples in the high temperature range. Glob. J. Res. Eng. Electr. Electron. Eng. 2013, 13, 7-12.

21. Poudel, B.; Hao, Q.; Ma, Y.; Lan, Y.; Minnich, A.; Yu, B.; Yan, X.; Wang, D.; Muto, A.; Vashaee, D.; et al. High-thermoelectric performance of nanostructured bismuth antimony telluride bulk alloys. Science 2008, 320, 634-638. [CrossRef] [PubMed]

22. Bubnova, O.; Khan, Z.U.; Matil, A.; Braun, S.; Fahlman, M.; Berggren, M.; Crispin, X. Optimization of the thermoelectric figure of merit in the conducting polymer poly(3,4-ethylenedioxythiophene). Nat. Mater. 2011, 10, 429-433. [CrossRef] [PubMed]

23. Kim, G.H.; Shao, L.; Zhang, K.; Pipe, K.P. Engineered doping of organic semiconductors for enhanced thermoelectric efficiency. Nat. Mater. 2013, 12, 719-723. [CrossRef] [PubMed]

(C) 2017 by the authors. Licensee MDPI, Basel, Switzerland. This article is an open access article distributed under the terms and conditions of the Creative Commons Attribution (CC BY) license (http:// creativecommons.org/licenses/by/4.0/). 